\title{
Integrated lipidomics and transcriptomic analysis of peripheral blood reveals significantly enriched pathways in type 2 diabetes mellitus
}

Chen Zhao ${ }^{1,2+}$, Jinghe Mao ${ }^{3 \dagger}$, Junmei $\mathrm{Ai}^{4}$, Ming Shenwu ${ }^{3}$, Tieliu Shi ${ }^{2}$, Daqing Zhang ${ }^{5}$, Xiaonan Wang ${ }^{1}$, Yunliang Wang ${ }^{6^{*}}$, Youping Deng ${ }^{1 *}$

From The 2011 International Conference on Bioinformatics and Computational Biology (BIOCOMP'11) Las Vegas, NV, USA. 18-21 July 2011

\begin{abstract}
Background: Insulin resistance is a key element in the pathogenesis of type 2 diabetes mellitus. Plasma free fatty acids were assumed to mediate the insulin resistance, while the relationship between lipid and glucose disposal remains to be demonstrated across liver, skeletal muscle and blood.

Methods: We profiled both lipidomics and gene expression of 144 total peripheral blood samples, 84 from patients with T2D and 60 from healthy controls. Then, factor and partial least squares models were used to perform a combined analysis of lipidomics and gene expression profiles to uncover the bioprocesses that are associated with lipidomic profiles in type 2 diabetes.
\end{abstract}

Results: According to factor analysis of the lipidomic profile, several species of lipids were found to be correlated with different phenotypes, including diabetes-related C23:2CE, C23:3CE, C23:4CE, ePE36:4, ePE36:5, ePE36:6; racerelated (African-American) PI36:1; and sex-related PE34:1 and LPC18:2. The major variance of gene expression profile was not caused by known factors and no significant difference can be directly derived from differential gene expression profile. However, the combination of lipidomic and gene expression analyses allows us to reveal the correlation between the altered lipid profile with significantly enriched pathways, such as one carbon pool by folate, arachidonic acid metabolism, insulin signaling pathway, amino sugar and nucleotide sugar metabolism, propanoate metabolism, and starch and sucrose metabolism. The genes in these pathways showed a good capability to classify diabetes samples.

Conclusion: Combined analysis of gene expression and lipidomic profiling reveals type 2 diabetes-associated lipid species and enriched biological pathways in peripheral blood, while gene expression profile does not show direct correlation. Our findings provide a new clue to better understand the mechanism of disordered lipid metabolism in association with type 2 diabetes.

\footnotetext{
* Correspondence: wangyunliang81@163.com; Youpingd@gmail.com

† Contributed equally

'Wuhan University of Science and Technology, Wuhan, Hubei 430081, P.R.

China

${ }^{6}$ Department of Neurology, The 148 Hospital of PLA, Zibo, Shandong,

255300, China

Full list of author information is available at the end of the article
} 


\section{Background}

Skeletal muscle and hepatic insulin resistance are key elements in the pathogenesis of type 2 diabetes mellitus (T2D) [1]. However, T2D is caused by not only insulin resistance [2], but also a heterogeneous cluster of conditions rather than a uniform entity [3]. Due to both environment and heredity heterogeneity, gene expression profiling is limited in exploring molecular mechanism of type 2 diabetes $[4,5]$.

As a comprehensive indicator, plasma free fatty acids were assumed to mediate the insulin resistance. Lipid profiling has already been applied in type 2 diabetes studies $[6,7]$, such as free fatty acids built linkage between the resistance and obesity [8]. However, the relationship between lipid and glucose disposal remains to be demonstrated across liver, skeletal muscle, and blood $[9,10]$. Here, we have integrated lipidomic analysis with gene expression profiling to discover the relationship between versatile lipid species and bioprocesses that are associated with type 2 diabetes. Using our model analysis, the statistically significant biological pathways were retrieved, and the findings provide a new strategy to link blood lipid species and illuminate the mechanism of insulin resistance associated with lipid and gene expression in blood.

\section{Results}

\section{Study subjects}

This study comprised a balanced distribution of the studied subjects in gender and race: among 60 controls, 28 were African American (AA) including 14 females and 14 males; 32 were Caucasian (CAU) including 14 females and 18 males. Among 84 patients with T2D, 44 were AA including 22 females and 22 males; 40 were CAU including 23 females and 17 males. As compared to AA, CAU had a significantly higher level of blood triglycerides (TG) in both the controls $(106 \pm 54.3 \mathrm{mg} / \mathrm{dl}$ in AA vs. $153 \pm 77.8 \mathrm{mg} / \mathrm{dl}$ in CAU, $\mathrm{p}=0.0009)$, and the patients

Table 1 The clinical characteristics of the study subjects

\begin{tabular}{lll}
\hline & $\begin{array}{l}\text { Normal controls } \\
(\boldsymbol{n}=\mathbf{6 0})\end{array}$ & $\begin{array}{l}\text { Diabetic } \\
(\boldsymbol{n}=\mathbf{8 4})\end{array}$ \\
\hline Age, yr (mean \pm SD) & $58.5 \pm 16.1$ & $63 \pm 13$ \\
Sex (female/male) & $28 / 32$ & $45 / 39$ \\
Race & 32 Caucasian & 40 Caucasian \\
& 28 African American & 44 African American \\
Body Mass index (kg/m) & $30.1 \pm 7.3$ & $34.2 \pm 8.4^{* *}$ \\
Triacylglycerol $(\mathrm{mg} / \mathrm{dl})$ & $134 \pm 76.9$ & $186 \pm 113.8^{* *}$ \\
HDL cholesterol $(\mathrm{mg} / \mathrm{dl})$ & $56.6 \pm 17.5$ & $49.1 \pm 15.4^{* *}$ \\
LDL cholesterol $(\mathrm{mg} / \mathrm{dl})$ & $112 \pm 44.8$ & $109.8 \pm 36.5$ \\
Total cholesterol (mg/dl) & $197 \pm 44.8$ & $195 \pm 46.8$ \\
Glucose $(\mathrm{mg} / \mathrm{dl})$ & $88.8 \pm 10.8$ & $142.7 \pm 56.8^{* * *}$ \\
\hline
\end{tabular}

Data are mean \pm SD. Statistical analyses were performed using the Student's t-Test. ${ }^{* *} p<0.05{ }^{* * *} p<0.001$, values are based on differences from controls.
$(157 \pm 128 \mathrm{mg} / \mathrm{dl}$ in AA vs. $207 \pm 98.3 \mathrm{mg} / \mathrm{dl}$ in CAU, $\mathrm{p}=0.037$ ). There were no significant differences in other studied clinical parameters between two races (data for racial differences were not shown). As compared to all controls (mixed), patient's group was 4.5 years older, had significantly higher body mass index (BMI), blood TG and fasting glucose, and lower high density apolipoprotein (HDL). There were no differences in low density apolipoproteins (LDL) and total cholesterols (Table 1) between controls and T2D patients.

\section{Plasma lipid profile reveals phenotype factors}

Plasma lipid profile is associated with various types of diseases or phenotypes. In order to illustrate the relationship between lipid species and gene expression level of peripheral blood, we performed unsupervised exploratory factor analysis and found significant linkages between lipid profile and phenotypes, including race, sex, and diabetes at the significant levels $1.87 \mathrm{e}-6,9.28 \mathrm{e}-$ 4, and 3.17 e- 3 by Wilcoxon Rank Sum Test, respectively. As shown in Figure 1, three types of CE species (C23:2CE, C23:3CE, C23:4CE) were found to be positively correlated with diabetes, while three types of ePE were shown to be negatively correlated. For sex, more than five and six lipid species were found to be correlated: PE40:5, PE36.4, and PE34.1 tend to be higher in female samples, while LPC18:2 and LCP18:1 were a little higher in male samples. For race, two types of SM (SM22:1 and SM22:0) were a little higher in black, while PE (PE34:2, PE36:3) and PI (PI36:1, PI38:3) were higher in white samples.

\section{Phenotype factors have lesser effect on gene expression profile}

Unlike the lipid profile, the gene expression profile does not show direct correlation with phenotype indicators, according to both a hierarchical clustering (Figure 2) and principal component analysis (PCA). As shown in the clustering, all of the data can be divided in to four main classes, but none of the factors (sex, diabetes, age, and race) were significantly correlated with main classes. However, race and sex were shown to be non-randomly distributed in the dendrogram, which implies underlying correlation with gene expression profile (GEP). Moreover, significant correlation was identified between GEP and phenotype factors based on PCA scores in the correlation test. GEP was correlated with race, and many genes may be differentially expressed between black and white samples. Race was the factor most known to be GEP-correlated, and tested as correlated with the third component ( $\mathrm{p}=7.20 \mathrm{e}-4$, Kruskal test $)$, which contains $5.8 \%$ variances. Diabetes was then tested to be correlated with the fifth component ( $p=8.67 \mathrm{e}-3$, Kruskal test), which contains $4.7 \%$ variances, and sex with the tenth component $(\mathrm{p}=$ 
$2.02 \mathrm{e}-2$, Kruskal test) containing $2.1 \%$ variances. There is almost $87.4 \%$ variance or unknown information in GEP. Direct differential expression genes were difficult to understanding in terms of biological meanings, which enriched in seemingly unrelated pathways (Table 2) such as ECM-receptor interaction and Riboflavin metabolism.

\section{Significant biological pathways link gene expression profile with lipid profile and diabetes}

To overcome the limitation of the unknown variances in gene expression profile, and to recover the relationship between gene expression profile and lipid profile, PLS regression model was adopted. A list of significant pathways from the gene expression profile was found to explain the lipid profiles, and also the lipid profile associated T2D (Table 3). Six of the top ten pathways have direct linkage with diabetes, including one carbon pool by folate, arachidonic acid metabolism, insulin signaling pathway, amino sugar and nucleotide sugar metabolism, propanoate metabolism, and starch and sucrose metabolism. None of them can be retrieved from a differential expression gene selection.

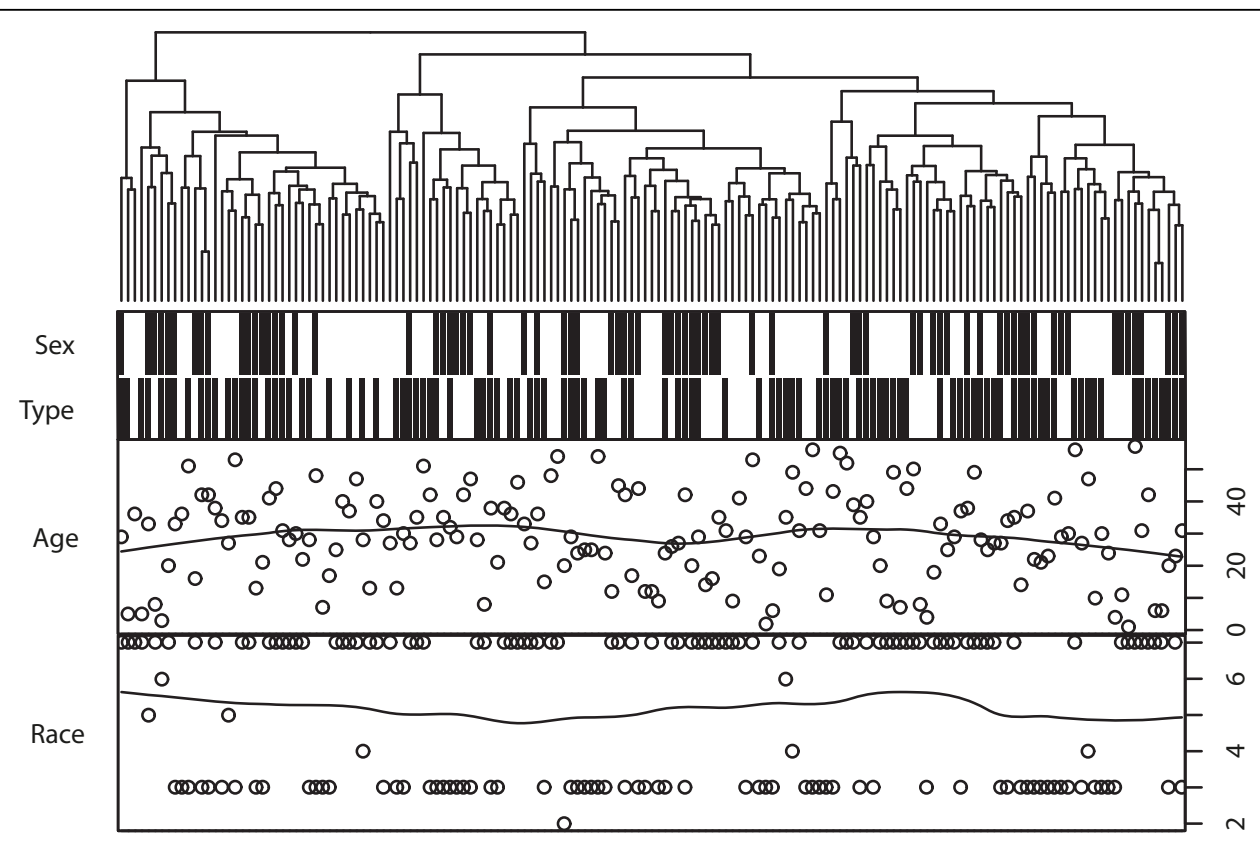

Figure 2 Hierarchical clustering of all samples in filtered data set. Factors such as sex and type were represented by black or white blocks: female was in white, male in black; diabetes in black, non-diabetes in white; Asian as 2, blacks as 3, Indian as 4, Mexican as 5 and 6, whites as 7. 
Table 2 Enriched pathways of differentially expressed genes.

\begin{tabular}{ccccccc}
\hline KEGGID & P-value & Odds ratio & ExpCount & Count & Size & Term \\
\hline $\mathbf{4 5 1 0}$ & $2.95 \mathrm{E}-05$ & 5.284093 & 2.478357 & 11 & 200 & Focal adhesion \\
$\mathbf{4 5 1 2}$ & $7.66 \mathrm{E}-05$ & 7.804973 & 1.053302 & 7 & 85 & ECM-receptor interaction \\
$\mathbf{7 4 0}$ & $2.81 \mathrm{E}-04$ & 31.125 & 0.13631 & 3 & 11 & Riboflavin metabolism \\
$\mathbf{5 1 4 6}$ & $9.78 \mathrm{E}-03$ & 4.162056 & 1.313529 & 5 & 106 & Amoebiasis \\
\hline
\end{tabular}

P-value is the significant level of KEGG pathway enrichment based on fisher exact test [16]. Odds Ratio and ExpCount are the corresponding Odds Ratio and expect observed number. Count is the number of differentially expressed genes in corresponding pathway, and size is the total gene number.

\section{Discussion}

Gene expression profiling was generally adopted for diabetes in the levels of cell lines and drug response $[11,12]$. Considering the environment and heredity heterogeneity, the homogeneity is not easy to conclude from a snapshot of the transcriptome for a wide cohort. Thus, we take lipid as an assistant to guide the exploration of gene-level mechanism of insulin resistance associated with lipid and gene expression in blood.

As expected, a major finding in our study is that very limited variance of transcriptome can be illustrated by the known phenotype factors. However, lipid profile shows an unexpected capacity on revealing the considered phenotype factors. By a lipid-guided exploration, a set of significant biological pathways and suspected genes were identified to be insulin resistance-associated, including one carbon pool by folate, arachidonic acid metabolism, and insulin signaling pathway, which cannot be directly found by gene expression profile. Our findings may prompt the understanding of the lipid associated gene-level mechanism of insulin resistance of type 2 diabetes mellitus in blood.

\section{Materials and methods}

\section{Subjects and clinical laboratory data}

The study was approved by the Institutional Review Board of Tougaloo College. All subjects provided written informed consent for this study. T2D was diagnosed based on American Diabetes Association (ADA) [5] and characteristic symptoms of diabetes, a higher BMI, and a fasting plasma glucose $>126 \mathrm{mg} \mathrm{dl}^{-1}$ or a $2 \mathrm{~h}$ plasma glucose during an oral glucose tolerance test of $>200$ $\mathrm{mg} \mathrm{dl}^{-1}$. A total of 144 blood samples from healthy controls ( $n=60,32$ Caucasians and 28 African Americans), and T2D ( $n=84,40$ Caucasians and 44 African Americans) were collected. All subjects were evaluated by age, sex, race, body mass index (BMI), triacylglycerol (TG), high-density lipoprotein (HDL), low-density lipoprotein (LDL), total cholesterol (TC), and glucose levels.

\section{Microarray experiments}

Total RNA from 8-10 mls peripheral blood WBCs was obtained using LeukoLock ${ }^{\mathrm{TM}}$ Total RNA system (Ambion Inc, Austin, TX) according to the manufacturer's instructions. The quantity and quality of the isolated RNA were evaluated by Nanodrop spectrophotometry and Agilent 2100 Bioanalyzer (Agilent Technologies, Santa Clara, CA). Gene expression profiling was peerformed using Agilent Whole Human Genome1 (4 X44K) Oligo arrays with 20,000 genes represented (Agilent Technologies, Palo Alto, CA). Each sample was hybridized with a human universal RNA control (Stratagene, La Jolla, CA). $500 \mathrm{ng}$ of total RNA was amplified and labeled using the Agilent Low RNA Input Fluorescent Linear Amplification Kit, according to manufacturer's protocol. For each two color array, $850 \mathrm{ng}$ of each Cy5- (universal control) and Cy3-labeled (sample) cRNA were mixed and fragmented using the Agilent In Situ Hybridization Kit

Table 3 Enriched pathways of differentially expressed genes

\begin{tabular}{|c|c|c|c|}
\hline Rank & KEGG Path ID & KEGG pathway name & Top 5 loadings gene \\
\hline 1 & path:hsa00670 & One carbon pool by folate & 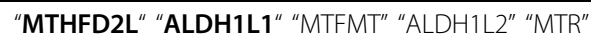 \\
\hline 2 & path:hsa00590 & Arachidonic acid metabolism & "PTGS2"'GPX7" "PLB1" "CYP4A11"'GPX2" \\
\hline 3 & path:hsa04910 & Insulin signaling pathway & "FLOT2" "PRKAB2"”MAPK8" "PPP1R3B" "PIK3CB" \\
\hline 4 & path:hsa05110 & Vibrio cholerae infection & "TJP2" "ATP6V1C1" "ADCY9"'ARF1" "ATP6V1H" \\
\hline 5 & path:hsa04020 & Calcium signaling pathway & "HTR4" "TRPC1"'ADCY8"'CHRM5"'GRIN2A" \\
\hline 6 & path:hsa00520 & Amino sugar and nucleotide sugar metabolism & "CHI3L1" "HK22"'NPL"“HEXA" "UAP1L1" \\
\hline 7 & path:hsa04062 & Chemokine signaling pathway & "CXCL5"'CXCL10" "PF4V1"'CCL8" "CXCL11" \\
\hline 8 & path:hsa00640 & Propanoate metabolism & "MLYCD" "ALDH2" "ACSS1" "PCCB"'ALDH3A2" \\
\hline 9 & path:hsa00500 & Starch and sucrose metabolism & "UGT2B17" "UGT2B15" "MGAM"'HK1" "AMY2B" \\
\hline 10 & path:hsa00240 & Pyrimidine metabolism & "DPYD"'ENTPD1"'TXNRD1"'"TK2" "TYMP" \\
\hline
\end{tabular}

Top Ten Enriched Pathways of most Factor Loading Genes. KEGG Path ID represents for KEGG pathway ID[ 19], which is ranked by pvalues of enrichment analysis. Pathways in gray lines have at least one bold marked gene, which is selected as a feature in gene based classifier. And the pathways in gray lines are the pathways that directly linked with diabetes $r$ by the marked genes. 
protocol. Hybridizations were performed for 17 hours in a rotating hybridization oven according to the Agilent 60-mer oligo microarray processing protocol prior to washing and scanning with an Agilent Scanner (G2565AA, Agilent Technologies, Wilmington, DE). Arrays were processed and background corrected with default settings for all parameters with the Agilent Feature Extraction software (v.9.5.3.1).

\section{Microarray data analysis}

Microarray data analyses were processed with GeneSpring version 7.0 and 10.0. The sample quality control was based on the Pearson correlation of a sample with other samples in the whole experiment. If the average Pearson correlation with other samples was less than $80 \%$, the sample was excluded for further analysis. More detailed analysis was done similar to previous description [13].

\section{ESI-MS/MS lipid profiling}

The same subjects that used for microarray experiments were also used for lipid profiling. Plasma was directly used for the lipid profiling, which was conducted as described previously [14].

\section{Statistical analyses}

To evaluate the correlation between various type of data and phenotypes, two-side Kruskal's test were performed in $R$ [15]. Pathway analysis of the expression data was performed by Fisher exact test with GOstats [16] package. Factor analyses of lipid profile were also preformed in R, where varimax rotation was used to seek a basis that most economically represents each individual. Feature selection and CSVM classifier were implement with CMA [17]. PLS regression model were built [18] with leave-one-out crossvalidation.

\begin{abstract}
Authors' contributions
YD initiated and oversaw the project. YD and MJ designed the study. MJ and MS conducted the experiments. YD. JA, ST, DZ and ZC performed the data analysis. YD and ZC drafted the manuscript. YD, ZC, DZ, XW and TS contributed to result interpretation and manuscript preparation.
\end{abstract}

\section{Competing interests}

The authors declare that they have no competing interests.

\section{Acknowledgements}

We thank Yan Li for carefully reading the manuscript. This study was supported by NIH/NCMHD/RIMI, P20MD002725.

This article has been published as part of BMC Medical Genomics Volume 6 Supplement 1, 2013: Proceedings of the 2011 International Conference on Bioinformatics and Computational Biology (BIOCOMP'11). The full contents of the supplement are available online at http://www.biomedcentral.com/ bmcmedgenomics/supplements/6/S1. Publication of this supplement has been supported by the International Society of Intelligent Biological Medicine.

\section{Author details}

${ }^{1}$ Wuhan University of Science and Technology, Wuhan, Hubei 430081, P.R. China. ${ }^{2}$ Center for Bioinformatics and Computational Biology, Shanghai Key
Laboratory of Regulatory Biology, the Institute of Biomedical Sciences and School of Life Science, East China Normal University, Shanghai 200241, China. ${ }^{3}$ Department of Biology, Tougaloo College, Tougaloo, MS 39174, USA. ${ }^{4}$ Department of Internal Medicine, Rush University Cancer Center, Rush University Medical Center, Chicago, IL 60612, USA. ${ }^{5}$ Center for Systems Biology, School of Mathematical Sciences, Soochow University, Suzhou, Jiangsu 215006, China. ${ }^{6}$ Department of Neurology, The 148 Hospital of PLA, Zibo, Shandong, 255300, China.

Published: 23 January 2013

\section{References}

1. Porte D Jr: Banting lecture 1990. Beta-cells in type II diabetes mellitus. Diabetes 1991, 40(2):166-180.

2. Bergman RN: Lilly lecture 1989. Toward physiological understanding of glucose tolerance. Minimal-model approach. Diabetes 1989, 38(12):1512-1527.

3. Savage DB, Petersen KF, Shulman GI: Disordered lipid metabolism and the pathogenesis of insulin resistance. Physiological reviews 2007, 87(2):507-520.

4. Frederiksen CM, Hojlund K, Hansen L, Oakeley EJ, Hemmings B, Abdallah BM, Brusgaard K, Beck-Nielsen H, Gaster M: Transcriptional profiling of myotubes from patients with type 2 diabetes: no evidence for a primary defect in oxidative phosphorylation genes. Diabetologia 2008, 51(11):2068-2077.

5. Sakoda H, Ogihara T, Anai M, Funaki M, Inukai K, Katagiri H, Fukushima $Y$, Onishi $Y$, Ono $H$, Yazaki $Y$, et al: No correlation of plasma cell 1 overexpression with insulin resistance in diabetic rats and 3T3-L1 adipocytes. Diabetes 1999, 48(7):1365-1371.

6. Shams ME, Al-Gayyar MM, Barakat EA: Type 2 diabetes mellitus-induced hyperglycemia in patients with NAFLD and normal LFTs: relationship to lipid profile, oxidative stress and pro-inflammatory cytokines. Scientia pharmaceutica 2011, 79(3):623-634.

7. Kashyap S, Belfort R, Gastaldelli A, Pratipanawatr T, Berria R, Pratipanawatr W, Bajaj M, Mandarino L, DeFronzo R, Cusi K: A sustained increase in plasma free fatty acids impairs insulin secretion in nondiabetic subjects genetically predisposed to develop type 2 diabetes. Diabetes 2003, 52(10):2461-2474.

8. Boden $G$, Shulman Gl: Free fatty acids in obesity and type 2 diabetes: defining their role in the development of insulin resistance and beta-cell dysfunction. European journal of clinical investigation 2002, 32(Suppl 3):14-23.

9. Bergman RN, Ader M: Free fatty acids and pathogenesis of type 2 diabetes mellitus. Trends in endocrinology and metabolism: TEM 2000, 11(9):351-356.

10. Bays H, Mandarino L, DeFronzo RA: Role of the adipocyte, free fatty acids, and ectopic fat in pathogenesis of type 2 diabetes mellitus: peroxisomal proliferator-activated receptor agonists provide a rational therapeutic approach. The Journal of clinical endocrinology and metabolism 2004, 89(2):463-478.

11. Das UN, Rao AA: Gene expression profile in obesity and type 2 diabetes mellitus. Lipids in health and disease 2007, 6:35.

12. Sreekumar R, Halvatsiotis P, Schimke JC, Nair KS: Gene expression profile in skeletal muscle of type 2 diabetes and the effect of insulin treatment. Diabetes 2002, 51(6):1913-1920.

13. Zhou X, Mao J, Ai J, Deng Y, Roth MR, Pound C, Henegar J, Welti R, Bigler SA: Identification of plasma lipid biomarkers for prostate cancer by lipidomics and bioinformatics. PLOS One 2012, 7(11):e48889.

14. Deng Y, Meyer SA, Guan X, Escalon BL, Ai J, Wilbanks MS, Welti R, GarciaReyero N, Perkins EJ: Analysis of common and specific mechanisms of liver function affected by nitrotoluene compounds. PLoS One 2011, 6(2): e14662.

15. Team RDC: R: a language and environment for statistical computing. 2008.

16. Falcon S, Gentleman R: Using GOstats to test gene lists for GO term association. Bioinformatics 2007, 23(2):257-258

17. Slawski M, Boulesteix AL, Bernau C: CMA: synthesis of microarray-based classification. 2009.

18. pls: Partial least squares and principal component regression. 2011.

19. Kanehisa M: The KEGG database. Novartis Foundation symposium 2002, 247:91-101, discussion 101-103, 119-128, 244-152. 
doi:10.1186/1755-8794-6-S1-S12

Cite this article as: Zhao et al:: Integrated lipidomics and transcriptomic analysis of peripheral blood reveals significantly enriched pathways in type 2 diabetes mellitus. BMC Medical Genomics 2013 6(Suppl 1):S12.

Submit your next manuscript to BioMed Central and take full advantage of:

- Convenient online submission

- Thorough peer review

- No space constraints or color figure charges

- Immediate publication on acceptance

- Inclusion in PubMed, CAS, Scopus and Google Scholar

- Research which is freely available for redistribution

Submit your manuscript at www.biomedcentral.com/submit 